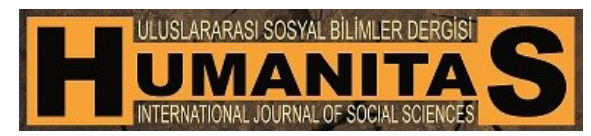

Humanitas,2018; 6(11): 129-147

ISSN:2147-0088X

\title{
ETHOS SEMI-AGENTIF : INTERVENTION DE SEGOLENE ROYAL SUR LES CENTRALES NUCLEAIRES
}

\author{
Ece KORKUT ${ }^{1}$ \\ Serap GÜL ${ }^{2}$
}

\begin{abstract}
Résumé : Un discours politique se distingue d'autres genres de discours par le double statut du locuteur qui représente moins luimême que son adhésion à une communauté. Dans cet article, il s'agit essentiellement de chercher la part de l'agentivité et de la responsabilité du locuteur. Le corpus analysé est une interview réalisée au journal télévisé de $20 \mathrm{H}$ de TF1 du 7 mars 2016, présenté par Gilles Bouleau avec Ségolène Royal, la ministre de l'environnement et de l'énergie. Le sujet de débat porte sur la fermeture éventuelle d'une centrale nucléaire en France, conformément aux engagements du Parti socialiste qui était au pouvoir. Bien qu'il s'agisse d'une interview, la méthodologie choisie n'est pas l'analyse conversationnelle, mais l'analyse du discours. Ce sont le dire et le dit de la ministre qui ont été analysés pour pouvoir révéler comment se construit son ethos discursif autour d'un problème écologique. Quoiqu'elle ait la légitimité de parole, de décision et d'action, la ministre se montre plutôt réticente devant une solution décisive. Au lieu de donner une réponse précise, elle opte pour une réponse conditionnelle et fortement modalisée, en délégant sa part de responsabilité à des tiers. C'est ainsi que son ethos discursif se manifeste comme quasi-agentif ou semi-agentif. Dans l'ensemble de cette interview, l'ethos discursif de SR se dessine de la manière suivante: éducateur, sceptique, hésitant, imprécis. Cet ethos qui se construit spontanément lors de son discours peut provenir aussi bien des interventions insistantes de l'interviewer que du positionnement incertain de son parti qui est au pouvoir.
\end{abstract}

Mots-clés : Discours politique, Analyse du discours, Ethos, Agentivité.

\footnotetext{
Prof. Dr., Hacettepe Üniversitesi, Fransız Dili Eğitimi Anabilim Dalı. ekorkut@hacettepe.edu.tr

2 Arş. Gör., Hacettepe Üniversitesi, Fransız Dili Eğitimi Anabilim Dalı. serapg@hacettepe.edu.tr
} 


\section{KONUŞAN ÖZNENIN KILICI ROLÜ : SEGOLENE ROYAL'IN NÜKLEER SANTRALLER KONUSUNDAKİ RÖPORTAJI}

Öz: Politik söylemi diğer söylem türlerinden ayıran en önemli özellik, konuşucunun ikili konumudur: Politik söylemdeki konuşucu kendine adına konuşmaktan çok, temsil ettiği grubun adına söz alır. Bu makalede esas olarak, konuşucunun kılıcı rolü ve sorumluluğu araştırılmıştır. Çözümlenen bütünce, 7 Mart 2016 tarihinde, Fransiz TF1 televizyon kanalındaki 20H Haber Programında gazeteci ve televizyoncu Gilles Bouleau'nun Fransa Çevre ve Enerji Bakanı Ségolène Royal ile gerçekleştirdiği bir röportajdır. Tartışma konusu, iktidardaki Sosyalist Parti'nin verdiği sözlerin gereği olarak, Fransa'daki bir nükleer santralin kapatılıp kapatılmayacağıdır. Karşılıklı konuşma söz konusu olduğu halde, çözümlemede kullanılan yöntem Konuşma Çözümlemesi değil, Söylem Çözümlemesidir. Bu çalışmada, bir çevre sorunu etrafinda oluşan söylemsel özsunumu ortaya çıkarmak amacıyla, bakanın söylem biçimi ve içeriği incelenmiştir. Bakan söz, karar ve eylem meşruiyetine sahip olsa da, kararlı bir çözüm önerme konusunda çekimser durmaktadır. Kendi sorumluluğunu daha çok üçüncü kişilere yükleyen bakan, kesin bir cevap vermek yerine koşullu ve kiplikli bir söylemi yeğlemektedir. Böylece yarı-kılıcı bir özsunum ortaya çıkmaktadır. Ele alınan röportajda S. Royal'in söylemsel özsunumu şu şekilde ortaya çıkmıştır: eğitimci, kuşkucu, kararsız, muğlak. Söylemi sırasında kendiliğinden oluşan bu özsunum, röportajı yapan gazetecinin 1srarlı tutumundan kaynaklanabileceği gibi, Çevre ve Enerji Bakanının bağlı olduğu ve iktidarda bulunan siyasi partinin konu ile ilgili olarak kararsız konumlanmasıyla da ilişkili olabilir.

Anahtar Sözcükler: Politik Söylem, Söylem Çözümlemesi, Özsunum, Kılıcıllk.

\section{Introduction}

Un discours politique est d'abord un " discours » qui est un ensemble langagier censé être assumé par son locuteur. Par cette propriété, le discours donne des informations -explicites ou implicites- sur les différents éléments constitutifs de l'identité et de l'ethos du locuteur. Ensuite vient le contenu «politique » qui est un domaine bien complexe $\mathrm{du}$ fait que le locuteur politique parle moins pour son compte qu'au nom du parti auquel il s'identifie ou qu'il représente. Il s'agit donc d'une part d'un locuteur singulier avec sa propre identité personnelle et de l'autre, d'un même locuteur qui est en demeure de s'exprimer en même temps au nom d'une collectivité, une collectivité qui change de positionnement, sinon d'identité, selon l'air du temps et les moments de crise. Ces deux 
identités, l'une personnelle, l'autre collective, se trouvent souvent imbriquées dans le cas d'un discours dit politique. Autrement dit, un locuteur a d'abord son propre habitus discursif, à quelle collectivité qu'il appartienne ; en outre, il doit être apte à adapter son discours personnel au positionnement actuel de cette identité collective. C'est dire que le locuteur d'un discours politique est là pour rapporter davantage les décisions et positionnements de son appartenance politique que ses propres choix politiques sur un sujet de débat précis.

Dans son article « Dire son identité politique », Damon Mayaffre (2003, p. 247) aborde les liens réciproques entre l'identité politique et le discours politique comme suit :

1) L'identité politique est toujours affaire de discours ;

2) Le discours politique est toujours affaire d'identité ou de stratégie identitaire.

Lorsqu'il apparaît une certaine incompatibilité entre l'identité personnelle et l'identité collective qui est circonstancielle et stratégique, cela donne lieu à des hésitations dans le discours.

Cet article traitera d'une interview réalisée avec la ministre de l'environnement et de l'énergie, Ségolène Royal ${ }^{3}$, à propos de la fermeture d'une centrale nucléaire en France. La ministre en question ayant un long passé politique a, de ce fait, un ethos préconstruit devant l'interviewer et le grand public. Nous ne révèlerons ici que l'ethos construit, ou l'image de soi, qui se dessine particulièrement dans cette interview. Comme l'affirme Maingueneau,

«ce que l'orateur prétend être, il le donne à entendre et à voir : il ne dit pas qu'il est simple et honnête, il le montre à travers sa manière de s'exprimer. L'ethos est ainsi attaché à l'exercice de la parole, au rôle qui correspond à son discours, et non à l'individu réel appréhendé indépendamment de sa prestation oratoire. » (1993, p. 138)

Notre point d'appui ne sera, par souci d'objectivité, que les éléments langagiers et discursifs (dont l'argumentation) réellement observables dans le discours de la ministre. Puisque parler, c'est à chaque fois une " construction discursive de l'identité » et la création d'ethos, " ou image discursive que l'orateur produit de sa propre personnalité » (Amossy, 2010, pp. 6, 16), nous allons chercher la construction identitaire de Ségolène Royal à travers ses interventions verbales. Par conséquent, nous essayerons d'analyser, dans un discours politique, la notion d'ethos discursif qui sera étudiée du point de vue du dire et du dit de

\footnotetext{
${ }^{3}$ Ministre de l'Environnement, de l'Énergie et de la Mer entre le 11 février 2016 et le 17 mai 2017.
} 
l'énonciatrice, et dans le cadre de l'agentivité et de la responsabilité du « je ».

\section{Le sujet de débat}

Dans l'interview d'une durée de 9' 57 , réalisée au journal télévisé de $20 \mathrm{H}$ de TF1 du 7 mars 2016 présenté par Gilles Bouleau (désormais GB), avec Ségolène Royal (désormais SR), le thème du débat est bien précis : " la promesse de fermer Fessenheim sera-t-elle tenue ?». L'interviewée est la ministre de l'environnement et de l'énergie qui est interrogée sur une question délicate et internationale, puisqu'il s'agit d'un grave risque de pollution, non pas uniquement régionale mais aussi globale. Cette promesse de fermeture de la centrale de Fessenheim effectuée par François Hollande lors de ses engagements à la présidentielle de 2012 s'inscrit dans le cadre de la demande de fermeture (puis de démantèlement) de la centrale nucléaire du Bugey située dans l'Ain à Saint-Vulbas, tout près de la Suisse (à $70 \mathrm{~km}$ de Genève). Cette demande vient justement des autorités suisses, et plus précisément de la ville et du canton de Genève, par l'intermédiaire de son maire, Mme Esther Alder. Les autorités genevoises ont décidé de se lancer, dès le 2 mars 2016, dans une « bataille juridique » en déposant une plainte auprès du Pôle-Santé du Tribunal de grande instance de Paris contre X ciblant la centrale du Bugey pour « mise en danger de la vie d'autrui et pollution des eaux ». Il est intéressant de noter que les intérêts helvètes sont représentés dans cette procédure par l'avocate et ancienne ministre française de l'environnement, Mme Corinne Lepage. De plus, les autorités suisses contestent le projet d'EDF (l'entreprise qui exploite les 58 réacteurs nucléaires sur le sol français et qui est détenue à $85 \%$ par l'Etat) de construire sur le site même du Bugey un centre de stockage de déchets nucléaires provenant des neuf réacteurs d'EDF en cours de démantèlement en France.

\section{Interventions de l'interviewer GB}

En dehors de l'ouverture et de clôture, GB et SR interviennent chacun 14 fois dont 3 sous forme de chevauchement. Avant d'analyser le discours de la ministre SR, il serait loisible de préciser les prises de parole de GB pour montrer le déroulement de l'interaction dialogale où les intervenants se positionnent l'un par rapport à l'autre. Les 14 prises de parole de l'interviewer se présentent comme suit :

1. Question $1:$ «(..) Allez-vous fermer cette centrale...?» (- Réponse de SR)

2. Renseignement complémentaire : «qui est la question des Suisses en fait $\gg-(\mathrm{SR})$ 
3. Renseignement complémentaire : «qui sont en fait des communes ou des regroupements de communes ouais $»-(\mathrm{SR})$

4. Question 1 renouvelée : «(...) Allez-vous fermer cette centrale nucléaire ou pas ?»-(SR)

5. Renseignement complémentaire : « ou la justice française » - (SR)

6. Correction et rappel : «entreprise EDF, c'est l'Etat, c'est à $85 \%$ c'est l'Etat »- (SR)

7. Question $2:$ « (...) fermer la centrale nucléaire de Fessenheim avant la fin du quinquennat. Est-ce que cela va être le cas ou pas ? » - (SR)

8. Question 2 renouvelée : «Ah, est-ce qu'elle va cesser de produire de l'électricité (...) ?» - (SR) [chevauchement]

9. Question 2 renouvelée: "Va-t-elle cesser de produire de l'énergie avant la fin du quinquennat de François Hollande ? » - (SR)

10. Rappel : «certes, mais l'engagement, l'engagement numéro 41 de François Hollande... » / (SR) / [chevauchement]

11. Question 2 renouvelée : « est de fermer. Cette promesse va-t-elle être tenue ?»-(SR : «OUI, elle sera tenue...»)

12. Mot phatique : «D'accord». - (SR)

13. Question $3:$ : Est-ce que vous allez autoriser cette augmentation des tarifs d'EDF ?» - (SR)

14. Mot phatique : «D'accord» - (SR) [chevauchement]

De ces interventions de GB ressort un certain nombre d'attitudes interactionnelles. GB ne pose en fait que trois questions liées dont la première a été posée 2 fois $(1,4)$ et la deuxième, 4 fois $(7,8,9,11)$. Cela parce que SR recourt aux différentes stratégies argumentatives -que nous verrons plus loin- qui l'éloignent du vif du sujet de débat. En effet, ce n'est qu'à la suite des interventions 10 et 11 de GB que vient «la» réponse de la part de SR («OUI »). Et la troisième question de GB en 13 apparait juste après ce «oui» obtenu difficilement, et qui implique un engagement («elle [cette promesse] sera tenue»). Il s'agit ici d'un engagement à propos d'un premier engagement (du parti socialiste). La troisième question, en relation avec la première et la deuxième, concerne une augmentation éventuelle du prix de l'électricité sur la demande « du patron d'EDF ».

A part ces trois questions, GB intervient trois fois pour apporter des informations supplémentaires ou complémentaires dans 2, 3, 5; une fois pour corriger dans 6, en rappelant le fait que EDF appartient à l'Etat ; une 
fois pour rappeler la responsabilité du gouvernement dans 10 ; et enfin, il utilise deux fois un mot phatique dans 12 et 14 : « d'accord ».

\section{Les traits langagiers et discursifs dans le discours de SR}

«Un discours politique, pour les linguistes, c'est du langage émis par une personne en direction d'une autre personne en vue de la convaincre. Dès lors les marques linguistiques du locuteur dans son texte, comme celles de l'auditoire, sont essentielles pour délimiter l'espace identitaire du discours. Qui parle? à qui ? Voici les deux principales questions, éminemment identitaires, directement abordables par l'étude des pronoms personnels, que pose tout discours politique. » (Mayaffre, 2003, p. 252)

Dans notre corpus, bien que les questions de l'interviewer soient assez claires et directes, on observe chez l'interviewée l'intention de développer le sujet de débat par des renseignements périphériques. Et pourtant, devant les insistances de l'interviewer, SR ne cède le pas par un « oui » que vers la fin de l'interview, en y ajoutant pourtant un « mais ». Par ailleurs, la manière de parler (le dire) a un effet incontestable sur le contenu du discours (le dit). Dans ce cadre, le discours de SR est caractérisé par des répétitions, ruptures, reprises de tournure et hésitations. On se contentera de ne citer ici que quelques exemples de répétitions et d'hésitations :

-répétitions (V. aussi 3.5.1.) : «bons sens » x 3 ; «modèle» x 6 ; «emplois» x 9 ; «ça veut dire quoi ? Ça veut dire qu'il faudra»; «il faut savoir une chose très simple, c'est très simple»; «Elles ont été difficiles à régir, ces règles dans la loi. Ça a été très difficile»; "l'énergie baisse, (...) le gaz baisse, le pétrole baisse »; "les antinucléaires, je leur dis, écoutez, (...) Les pronucléaires, je leur dis non »

-hésitations: " je vais pouvoir débloquer, le gouvernement va débloquer»; «L'Etat lui a dit et le parlement lui a dit»; «Aujourd'hui, les baisses, l'énergie, le prix de l'énergie baisse »

\subsection{Poser des questions pour y répondre}

SR pose des questions pour y répondre immédiatement elle-même. Cette attitude semble attribuer à la locutrice un rôle d'éducateur qui se cristallise par des actes tels que questionner, répondre, expliquer, expliciter («Il y a deux choses dans le mot fermeture »; «Il y a deux conditions pour qu'elle s'arrête définitivement»). En voici quelques exemples :

«Est-ce que l'on peut remplacer 10 réacteurs uniquement par des énergies renouvelables? La réponse est non. »

«Est-ce que c'est cela que l'on veut? Non. » 
« Ça veut dire quoi ? Ça veut dire qu'il faudra... »

«Quel est-il ? C'est très simple. »

«Et qu'est-ce que l'on observe depuis deux années, depuis le début de ce débat? C'est que les installations d'énergie photovoltaïques solaires ont augmenté de $40 \%$ en deux ans. »

«Comment? D'une part en fermant les centrales...»

\subsection{Vous (GB) et Je (SR) - Les marqueurs modaux}

Selon Amossy (2010, p. 10),

«l'investigation des discours en « je» (...) permet de voir comment l'identité se construit dans l'échange verbal, comment elle se négocie dans le rapport à l'autre, en quoi elle est liée à des questions d'efficacité discursive. (...) Et la gestion de l'ethos est toujours collective ».

Avant d'étudier les syntagmes où apparaît le « je » de SR, signalons que GB adresse la parole à SR directement par un «vous » singulier et agentif; «vous» en tant que ministre de l'environnement et de l'énergie :

«Vous êtes la ministre de l'environnement et de l'énergie. »

«Allez-vous fermer cette centrale qui a été construite je crois en 1978 ? »

«Allez-vous fermer cette centrale nucléaire ou pas?»

« Le patron d'EDF réclame à corps et à cri, il vous réclame à vous une augmentation des tarifs d'EDF. Est-ce que vous allez autoriser cette augmentation des tarifs d'EDF? »

Par contre, le «je ${ }^{4}$ de SR est la plupart du temps accompagné de marqueurs modaux. «Dire 'je', c'est marquer sa singularité... Dire 'je' dans le discours politique, c'est refuser son identité de locuteur politique, de porte parole des autres par exemple, de représentant d'une classe, d'un parti, d'une nation... » (Mayaffre, 2003, p. 253). Le « je » agentif de SR ne se manifeste explicitement que quand il est question du contenu de sa mission dont les frontières ont été tracées par elle-même : défendre et appliquer le modèle français, créer des emplois, faire voter la loi, poser une condition. Ces exemples peuvent être considérés comme une affirmation pour asseoir sa légitimité et pour circonscrire les domaines de sa responsabilité :

« je suis là pour défendre ce modèle français, pour l'appliquer avec bon sens, détermination et en créant le plus d'emplois possible. » ;

\footnotetext{
${ }^{4}$ Dans l'ensemble de l'interview, nous avons relevé 25 marqueurs renvoyant au /JE/ : ‘je', 'j' $=23$; 'mon' $=1 ;$ 'me' : 1 .
} 
« la loi que j'ai fait voter»;

« Et la deuxième condition, ça, c'est moi qui la donne ».

Quant aux autres actes que réalise ou rapporte la ministre dans son discours avec le « je », ils sont les suivants : observer, annoncer, rappeler, conseiller, dire, demander :

«j'observe d'ailleurs qu'il y a un débat démocratique »;

« le gouvernement, je vous l'annonce, vient de décider... »;

«ce crédit d'impôt qui, je le rappelle, permet de rembourser $30 \%$ des dépenses »;

« un site que je lui [à Tesla] conseillerais volontiers pour justement rebondir vers le haut et fermer en effet définitivement Fessenheim. »;

«j'ai dit à nos amis allemands (...) vous allez aussi nous aider à faire la mutation de ce site $»$;

« je demande à EDF de faire d'abord des progrès de productivité ».

Il nous faut ajouter aussi que tout au long de son discours, SR adresse la parole non seulement à l'interviewer, mais aussi à des tiers tels que les Suisses, les antinucléaires, les pronucléaires, les Français et Tesla :

« il faut dire aux Suisses »;

« les antinucléaires, je leur dis, écoutez »;

«Les pronucléaires, je leur dis non »;

« Moi, je voudrais quand même dire aux Français qui nous écoutent »;

« je lui [à Tesla] conseillerais volontiers ».

Les marqueurs de modalité dans le discours de SR, ici réalisée par le « je », se regroupent autour des modalités volitive et épistémique. La modalité volitive, donc subjective, qui est en rapport avec le désir et la volonté, s'actualise dans les énoncés suivants :

«je voudrais dire [...] que la France est en train de rattraper»;

« Moi, je voudrais quand même dire aux Français »;

«Moi, je veux que la facture des Français baisse »;

«Moi aussi, je rêverais que toutes les énergies en France soient des énergies renouvelables ».

Ces séquences d'énoncés qui traduisent une certaine volonté de SR sont souvent suivies de l'expression d'un obstacle réel qui rend impossible la réalisation de cette volonté personnelle.

Le deuxième type de modalité qui se manifeste dans le discours de SR, c'est la modalité épistémique. Rappelons que «les modalités épistémiques marquent le domaine du certain, du douteux, du savoir, de la croyance » (Le Querler, 1996, p. 41). On considère comme des 
marqueurs épistémiques « des verbes comme savoir, croire, des adverbes comme peut-être, sans doute, probablement, des tiroirs verbaux en -rais dans certains de ses emplois » (idem, p. 55).

« je crois que sur ces sujets-là, il faut se garder...»;

« je crois que la hausse n'aura pas vraiment lieu...»;

«moi, je crois que sur un site comme celui-ci, on peut effectivement construire...»; « la hausse, à mon avis, devra être de $0,5 \%$ »;

«qu'il faudra en effet fermer les centrales les plus anciennes, peut-être Bugey, mais...»

En outre, la temporalisation dans les énoncés de SR porte également une modalisation. Pour n'en donner que quelques exemples :

«je vais pouvoir débloquer $(. .$.$) les moyens de faire des travaux d'économie$ d'énergie »; (probabilité)

«moi aussi, je rêverais que toutes les énergies en France soient des énergies renouvelables »; (souhait)

« un site que je lui conseillerais volontiers pour justement rebondir vers le haut et fermer en effet définitivement Fessenheim » (souhait).

Il nous faut indiquer également que les énoncés de SR sont fortement marqués par divers types de modalités, et cela non seulement dans des énoncés où figure le « je » (V. 3.3.3., modalité déontique).

\subsection{Extension du « je » : Les tiers qui remplacent le « je » agentif}

Par ailleurs, lorsqu'il s'agit d'une prise de responsabilité proprement dite, le « je » est souvent remplacé par les identités communes ou les autorités autres. Dans d'autres cas, le sujet plein cède sa place à l'impersonnel ou à des emplois sans personne avec la passivation.

\subsubsection{Nous}

Le «nous», en tant qu'une extension du « je », se manifeste en : « je + tu » (le nous inclusif), « je $+\mathrm{il}(\mathrm{s}) »$ (le nous exclusif) ou encore « je + tu + il(s) ». Comme le précise Amossy (2010, p. 159), «l'ethos des discours en 'nous' témoigne (...) de la façon dont le moi s'étend et s'amplifie pour offrir une image de groupe », ou encore pour partager la part de responsabilité avec des tiers.

«Le 'nous' est fondamentalement politique (c'est même pour les analystes le premier mot politique de la langue française) et d'évidence identitaire : il a pour vocation, dans la langue politique, de construire une identité collective, de constituer une communauté, d'élever une pensée privée au statut de pensée ou de discours public, de substituer à 
l'individualité du locuteur l'identité plurielle politique du groupe. (Mayaffre, 2003, pp. 251-252).

Il apparaît dans le discours de SR 6 signes qui réfèrent au 'nous collectif' (4 « nous », 1 « nos », 1 « notre »). A l'exception d'un « nous » qui ne renferme que l'interviewer et l'interviewée («Je voudrais (...) dire aux Français qui nous écoutent... »), tous les emplois ont des référents plus moins précis dont également fait partie le « je » :

« le socle de notre modèle énergétique $»=$ le modèle français.

«nous devons passer de $75 \%$ de nucléaire (...) à $50 \%$ » = le gouvernement.

«nous allons accélérer les créations d'emploi dans cette filière.» $=$ le gouvernement.

« j'ai dit à nos amis allemands [...] vous allez aussi nous aider à faire la mutation de ce site $»=$ la France.

«Raisonnons avec bon sens et pragmatisme » = vous, moi et les autres.

\subsubsection{On}

Contrairement au « nous », le référent de tous les emplois du « on » se manifeste comme «le gouvernement» auquel appartient SR. Il s'agit donc d'une identité collective :

«on peut effectivement construire des installations d'énergie renouvelable, on peut faire une opération exemplaire de démantèlement. »

« et on peut installer d'autres usines »

«Donc on doit diversifier le mixte énergétique. »

«on a fait une réforme qui a freiné le prix de l'électricité. »

« on estime le potentiel de création d'emplois dans ces énergies vertes à 100000 emplois »

« est-ce que l'on peut remplacer 10 réacteurs uniquement par des énergies renouvelables? La réponse est non. »

«on va pas fermer du jour au lendemain les centrales »

Ce réfèrent unique a une seule exception dans le discours de SR. C'est l'énoncé : « ou alors y'a plus d'électricité, y'a plus d'usine qui fonctionne, est-ce que c'est cela que l'on veut? Non. » Dans cet énoncé, le « on » signifie « eux ; les antinucléaires ».

\subsubsection{Impersonnel}

Les emplois impersonnels se manifestent dans le discours de SR en « il faut » et « il va falloir » qui figurent 7 fois au total. Il s'agit là d'une modalité déontique qui renferme [la permission (pouvoir) et] l'obligation (devoir, falloir). Par ailleurs, cette formule en impersonnel qui revient 
souvent dans le discours de SR semble signaler une intention d'effacer le sujet agentif. Ce qui conduit à un choix discursif calculé.

« il faut dire aux Suisses [...] Donc la France a fait son choix. »;

« il faut se garder et des polémiques politiciennes et d'opposer les énergies les unes aux autres »;

« il faut savoir une chose très simple, c'est très simple »;

« il faut faire preuve de bon sens »;

« il faut d'ailleurs profiter de ces baisses pour investir dans les énergies

renouvelables »;

« il va falloir en effet prendre des décisions qui permettent d'appliquer euh,

d'appliquer cela. »;

« il va falloir réorienter les investissements du nucléaire vers le renouvelable. »

\subsubsection{Passivation sans agent}

La passivation, on le sait, est l'un des procédés de thématisation. Ce qui est à remarquer dans les formules passivées, utilisées souvent par SR, c'est que l'agent du procès n'est jamais exprimé. Ainsi, dans les exemples suivants, quand l'agent n'est pas précisé par le contexte, l'auditeur se posera la question «par qui ? ». Il s'avère que cet effacement de l'agent est un choix délibéré fait par la ministre :

« ce sont 8000 emplois qui y ont été crées » $(\mathrm{y}=$ dans la filière d'installation des doubles fenêtres) »;

« les 250 millions d'euros qui ont déjà été donnés à ces territoires d'énergie positive sont renouvelés »;

« les autorisations de centrales ont été données pour 40 années »;

« c'est pas le choix qui a été fait il y a 40 ans »;

« Elles ont été difficiles à régir, ces règles dans la loi. »;

«ce retrait de l'autorisation de fonctionner sera fait lorsque le conseil d'administration au mois de juin en aura fait la demande » ;

« oui, elle [cette promesse] sera tenue, je viens de vous le dire. »;

« la hausse, à mon avis, devra être de $0,5 \%$, mais pas plus, en fonction justement de la réforme qui a été faite pour maitriser une hausse... ».

\subsection{Implicite}

« Le décodage des contenus implicites » éventuels, c'est-à-dire ce qu'on dit indirectement, nécessite un «calcul interprétatif» et exige des compétences non-linguistiques (Kerbrat-Orecchioni, 1998, pp. 8-9). En effet, « une étude de l'implicite nécessite que l'on examine à la fois le support linguistique qui le rend possible et les compétences encyclopédiques ou le savoir partagé qui autorisent son déchiffrement » 
(Amossy, 2012, p. 192). En outre, le recours à l'implicite peut provenir de l'intention d'écarter une contre-réaction. «Une (...) origine possible au besoin d'implicite tient au fait que toute affirmation explicitée devient, par cela même, un thème de discussion possible. Tout ce qui est dit peut être contredit » (Ducrot, 1972, p. 6).

Nous allons citer ici quelques emplois implicites dans le discours de SR et les interpréter. Dans la séquence suivante, on valorise l'atmosphère actuelle créée par le pouvoir du PS par l'énoncé « il y a un débat démocratique » qui vient «enfin». Cet implicite est précisé dans la phrase suivante qui peut se résumer comme suit : avant notre pouvoir, il n'y avait pas de débat démocratique et les Français n'étaient pas informés sur ce qui se passait :

«A toutes ces questions sur le nucléaire, j'observe d'ailleurs qu'il y a un débat démocratique et que enfin les Français sont informés de ce qui se passe sur le nucléaire, ce qui n'a pas été le cas lorsque des décisions ont été prises. »

Par ailleurs, dans l'un des emplois de « bon sens », SR appelle les Suisses et tous les antinucléaires à faire preuve de bon sens (comme elle). Ainsi, elle sous-entend que ces derniers en manquent :

« il faut faire preuve de bon sens aussi, vous savez, moi aussi, je rêverais que toutes les énergies en France soient des énergies renouvelables, c'est pas le choix qui a été fait il y a 40 ans »

Enfin, lorsque SR pose la France comme la seule décideuse en la question, elle sous-entend que les Suisses ou autres n'ont pas le droit d'y intervenir :

«C'est la France qui décide de son modèle énergétique » (et personne d'autre).

\subsection{Argumentation}

« Argumenter, c'est adresser à un interlocuteur un argument, c'est-à-dire une bonne raison, pour lui faire admettre une conclusion et l'inciter à adopter les comportements adéquats » (Plantin, 1996, p. 24).

Dans son Argumentation dans la Communication, Philippe Breton (2006, p. 42) précise que les arguments que nous pouvons avancer pour défendre une opinion peuvent se classer en quatre grandes familles :

- Les arguments de cadrage

- Les arguments d'analogie

- Les arguments d'autorité

- Les arguments de communauté

Chaque grande famille contient des types d'arguments bien précis. Dans ce sens, par exemple, nous verrons pour les arguments de cadrage qu'ils peuvent avoir recours à la définition, la présentation, l'association ou la 
dissociation. Les arguments d'analogie auront recours à l'analogie proprement dite, l'exemple et la métaphore. Les arguments d'autorité à la compétence, l'expérience et le témoignage et enfin, les arguments de communauté à l'opinion commune, les valeurs et les lieux (Breton, 2006, p. 45).

Voici ci-dessous les types d'arguments dont a fait usage Ségolène Royal pour défendre son opinion.

\subsubsection{Arguments de cadrage}

Les arguments de cadrage consistent à présenter le réel sous un certain angle de manière à amplifier certains aspects de l'objet de discussion et en minorant d'autres dans le but de faire ressortir la légitimité de son opinion. Une forme d'argument de cadrage particulièrement puissante et dont la ministre fait usage est la présentation des faits de façon à majorer certains aspects et à en minorer d'autres. Par exemple, la ministre :

- présente le nucléaire sous son aspect de nécessité pour la production électrique en minorant l'aspect dangereux et polluant de ses déchets radioactifs ;

- insiste sur la création d'emploi dans les travaux d'économie d'énergie et d'équipement en énergie renouvelable ;

- affiche le débat auquel elle a été confrontée avec les antinucléaires et les pronucléaires.

L'amplification comme argument de cadrage est également très utilisée par Ségolène Royal. «Pour créer la présence, il est utile d'insister longuement sur certains éléments qui ne sont pas douteux : en prolongeant l'attention qu'on leur accorde, on augmente leur présence dans la conscience des auditeurs (Perelman, 1988, pp. 51, 52 cité par Breton, 2006, p. 83). Cette insistance peut être assurée par la répétition, l'accumulation des détails et l'accentuation de certains passages. Ainsi, l'amplification montre l'une des positions stratégiques pour défendre une opinion. On observe en effet dans le discours de SR une amplification constante qui constitue une forme convaincante d'arguments.

Dans cette perspective, voici les propos de la ministre SR qui s'inscrivent dans ce type d'arguments :

-Répétitions des structures syntaxiques :

« qui a été adoptée, qui a été votée, qui a été débattue »;

« je vais pouvoir débloquer, le gouvernement va débloquer »;

«une branche de l'entreprise qui fait des bénéfices, qui fait beaucoup de bénéfices »; 
« c'est 2000 emplois, c'est 2000 personnes, c'est 2000 salariés ».

-Accentuation de la séquence sur le modèle français qui donne la priorité aux énergies renouvelables, sur le montant des aides gouvernementales pour la transition énergétique :

« la loi que j’ai fait voter dit enfin que les énergies renouvelables sont une priorité »;

« 8000 euros pour une personne seule, 16000 euros pour un couple ».

-Accumulation des détails sur le pourcentage des énergies dans la production électrique en relation avec les années et le nombre d'emplois.

«nous devons passer de $75 \%$ de nucléaire aujourd'hui pour produire de l'électricité à 50\% à l'horizon $2025 »$;

« cela a créé 10000 emplois et on estime le potentiel de création d'emplois dans ces énergies vertes à 10000 emplois ».

L'intention de donner trop de précisions peut mener à l'imprécision ou révéler une position consistant à détourner le thème du débat vers des sous-thèmes ou des thèmes périphériques, dans l'espoir d'ouvrir d'autres sujets de débat. Cette attitude de l'interviewé(e) lui permet parfois de brouiller les pistes, fût-ce pour un moment. Dans un tel cas, l'interviewer a deux voies devant lui : soit il se fait avoir et poursuit la nouvelle piste ouverte, soit il obstine et revient constamment sur son thème initial. Dans notre cas, GB a opté pour cette dernière position.

Enfin, un autre type d'arguments de cadrage est la dissociation qui est une " méthode de cadrage du réel qui, à partir d'une notion qui renvoie habituellement à un seul et même univers, permet de le casser et de générer deux univers distincts » (Breton, 2006, p. 90). Lorsque SR dit "Y'a deux choses dans le mot fermeture », elle fait exactement usage d'une dissociation de cette notion en deux éléments. Le premier implique, selon la ministre, le retrait de l'autorisation de fonctionner et la seconde, même si la ministre ne le dit pas ouvertement, implique la fermeture effective, puis le démantèlement de la centrale.

Ce choix d'une notion globale, en l'occurrence fermeture, est essentiellement argumentatif, comme l'affirme Philippe Breton (2006, p. 91), car il ne s'appuie sur aucune classification scientifique.

\subsubsection{Arguments d'analogie}

Les arguments d'analogie servent à mettre en œuvre des figures classiques, comme l'exemple, l'analogie proprement dite ou la métaphore, en leur ajoutant une portée argumentative. L'opinion que l'on défend dans ce cas est appelée thème et l'élément extérieur qui sert à composer l'argument est appelé phore. Autrement dit, « on appelle phore la relation déjà admise $(. .$.$) et thème la relation à faire admettre (. .$.$) , ce$ 
qui équivaut aux termes de comparant (= phore) et de comparé (= thème) » (Robrieux, 2010, p. 203) dans le cas de la métaphore.

L'analogie proprement dite :

- Lorsque la ministre évoque la fermeture des centrales qui ne correspondent plus aux normes de sécurité, elle fait une analogie avec la centrale nucléaire de Fukushima.

- Lorsque la ministre dit « fermer un réacteur ou une centrale, c'est pas tourner un robinet», elle fait également une analogie avec un élément extérieur dont le thème est la fermeture de la centrale et le phore est la fermeture du robinet. Comme on le constate dans cet exemple, le phore « est en général pris dans le domaine du sensible, concret » et «le thème est en général abstrait» (Reboul, 1991, p. 189).

\subsubsection{Arguments d'autorité}

Les arguments d'autorité recouvrent tous les procédés qui permettent de mobiliser une autorité acceptée par l'auditoire. Cette autorité, dans l'argument, est censée ainsi défendre l'opinion que l'on propose ou que l'on critique. La forme de ces arguments est donc constante : l'opinion que l'on défend devient acceptable par l'auditoire car l'autorité la soutient et cette autorité est reconnue par l'auditoire. De plus, avec les arguments d'autorité, nous avons affaire à une délégation de savoir comme par exemple, lorsque SR dit «c'est la France qui décide de son modèle énergétique ", elle délègue son savoir à la France qui sait mieux qu'elle le modèle énergétique que l'on doit adopter.

Tout au long de son intervention, SR invoque des entités tierces en substantif dans le but justement de faire appel aux arguments d'autorité et de déléguer son savoir. Nous l'avons vu plus haut, les arguments d'autorité peuvent se différencier selon qu'ils font appel à la compétence ou à l'expérience. Ainsi, nous pouvons classer ces entités tierces en fonction de cela et dire que «la loi, le gouvernement, le parlement, la France, l'Etat» entrent dans la catégorie des arguments d'autorité par expérience et que «EDF et l'Autorité de Sûreté nucléaire » entrent dans celle des arguments d'autorité par compétence. Voici les propos de la ministre en ce sens :

«c'est à l'entreprise EDF de dire quelle centrale il veut fermer »;

« la loi donne à EDF la responsabilité de fermer ces centrales »;

« autorité de sûreté nucléaire qui donne les autorisations de prolongation »;

« la loi française qui vient d'être adoptée, qui a été votée, qui a été débattue par la droite, par la gauche »; 
« la France a fait son choix souverain de modèle énergétique »;

« le gouvernement vient de décider que... »;

« l'Etat lui a dit et le parlement lui a dit qu'il fallait baisser la part du nucléaire ».

Ainsi, nous voyons que lorsque SR invoque dès le début de son intervention « la loi française » pour tenter de répondre à la question de $\mathrm{GB}$, elle fait appel à l'autorité que représente la loi. De même, en invoquant par la suite et à plusieurs reprises le gouvernement, le parlement, la France, l'entreprise EDF et l'Autorité de Sûreté nucléaire, elle se réfère à ces autorités pour déléguer sa part de responsabilité dans la décision finale de fermeture de la centrale nucléaire.

\subsubsection{Arguments de communauté}

Enfin, les arguments de communauté font appel « à des croyances ou à des valeurs partagées par l'auditoire, qui contiennent déjà, en quelque sorte, l'opinion qui est l'objet de l'entreprise de conviction » (Breton, 2006, p. 42, 43). Ces arguments s'appuient sur des valeurs qui sont ainsi censées être partagées par beaucoup. Invoquer « le modèle français, les Français, le savoir-faire français », comme la ministre le fait dans les propos suivants, consiste à utiliser un argument de ce type pour capter l'attention de son auditoire sur ces aspects de l'objet de discussion :

« les Français ont payé pendant quarante ans ces centrales nucléaires »;

« c'est un marché pour EDF et pour le savoir-faire français »;

« la France est en train de rattraper son retard».

\section{Conclusion}

Le fait de représenter simultanément deux identités dans un même discours ou dans des discours successifs pose souvent un problème sur le plan de l'ethos, donc un problème éthotique. C'est bien le cas pour les preneurs de décision dans le domaine de la politique.

Un même locuteur peut en effet construire, consciemment ou non, des ethos différents dans des situations différentes. Dans le cadre de cette interview analysée du point de vue de quelques éléments discursifs et argumentatifs, nous avons révélé les points suivants :

- L’abondance des énoncés modalisés montre que SR n'opte pas pour une expression directe, nette et prometteuse. Cette attitude discursive donne à ses propos un ton indécis. Les énoncés volitifs et épistémiques (en doute et en croyance) escamotent une assertion décisive. En outre, les hésitations et les répétitions fréquentes (le dire) qui apparaissent dans l'immédiat du discours oral et spontané viennent rendre plus manifeste cette imprécision dans son discours de manière à le transformer en une indécision. 
- Quand il s'agit de prendre une responsabilité résolue pour son compte, l'extension du «je» vers d'autres pronoms («nous», « on ») et autorités laisse entendre que SR délègue souvent les questions épineuses à des tiers tels que l'Autorité de Sûreté nucléaire, EDF, etc. Dans un tel cas, c'est l'agentivité du «je » de la ministre qui est mise en jeu. Il faut le considérer comme une stratégie discursive, effectuée de la part de la ministre. Bien qu'elle ait la légitimité de parole, de décision et d'action, la ministre se montre donc plutôt réticente devant une réelle solution à un problème international.

- L'interviewée argumente ses propos en se référant à des procédés argumentatifs bien divers comme l'amplification, l'analogie, l'argument d'autorité et l'argument de collectivité... Ce qui s'expliquerait par une certaine intention de chercher des prétextes à son indécision apparente.

- La ministre interviewée a tendance à adopter un positionnement plutôt neutre dans son discours. Vers la fin de son intervention, elle dit «non» aux deux parts, antinucléaires et pronucléaires, car selon elle, des deux parts il y a un risque et il faut de ce fait se comporter «avec bon sens». A la suite de nombreuses hésitations, ses arguments qui touchent les réalités évidentes et les risques apparents semblent ici plus pertinents :

«Les antinucléaires, je leur dis, écoutez, on va pas fermer du jour au lendemain les centrales ou alors y'a plus d'électricité, y'a plus d'usine qui fonctionne, est-ce que c'est cela que l'on veut? Non. Les pronucléaires, je leur dis non, ne dites pas que le nucléaire est une énergie propre puisqu'il y a le problème du déchet, y'a le problème de la sûreté. »

Néanmoins, dire «non » aux deux parties prenantes (anti et pro) ne contribue pas à une solution qui puisse être exprimée avec détermination.

- Dans l'ensemble de cette interview, l'ethos discursif de SR se dessine de la manière suivante : éducateur, sceptique, hésitant, imprécis. Cet ethos qui se construit spontanément lors de son discours peut provenir aussi bien des interventions insistantes de l'interviewer que du positionnement incertain de son parti qui est au pouvoir.

Pour pouvoir donner du sens à un discours politique spontané, force est de connaître et de prendre en compte toutes les circonstances d'échange, la position et le positionnement actuel du locuteur ainsi que les 
dimensions sociopolitiques du thème abordé. A la fin de cette analyse du discours succincte, reste à savoir si cet ethos qui se construit appartient plutôt à la personne singulière qu'est Ségolène Royal, ou bien au parti qu'elle est en demeure de défendre d'une manière ou d'une autre. Posons la question avec Amossy, elle-même liée à l'agentivité et à la responsabilité : « le locuteur projette-t-il son image de soi, comme le veut la rhétorique, en véritable agent qui agit et prend des responsabilités dans les affaires humaines ou, comme le suggèrent la microsociologie et l'analyse du discours, en sujet modelé par les modèles et les routines de sa communauté ?» (Amossy, 2010, p. 41). Si cet article a atteint son objectif, la réponse s'avère dans la deuxième partie de la question.

\section{Bibliographie}

Amossy, R. (2010). La Présentation de soi. Ethos et identité verbale. Paris : PUF.

Amossy, R. (2012). L'argumentation dans le discours. Paris : Armand Colin.

Breton, P. (2006). L'argumentation dans la communication. Paris : Editions La Découverte.

Ducrot, O. (1972). Dire et ne pas dire. Principes de sémantique linguistique. Paris : Hermann.

Kerbrat-Orecchioni, C. (1998). Implicite ( $2^{\mathrm{e}}$ édition). Paris : Armand Colin.

Lamizet, B. (2011). Le langage politique. Paris : Ellipses.

Le Querler, N. (1996). Typologie des modalités. Caen: Presses universitaires de Caen.

Maingueneau, D. (1993). Le Contexte de l'œuvre littéraire. Enonciation, écrivain, société. Paris : Dunod.

Mayaffre, D. (2003). «Dire son identité politique ». Cahiers de la Méditerranée. [En ligne] 66 | 2003, mis en ligne le 21 juillet 2005, consulté le 11 avril 2016. URL : http://cdlm.revues.org/119

Plantin, C. (1996). L'Argumentation. Paris : Le Seuil. Mémo.

Reboul, O. (1991, 2013). Introduction à la rhétorique. Paris : PUF.

Robrieux, J.-J. (2010). Rhétorique et argumentation ( ${ }^{\mathrm{e}}$ édition). Paris : Armand Colin. 


\title{
SEMI-AGENTIVE ETHOS: INTERVIEW OF SEGOLENE ROYAL ON NUCLEAR PLANTS
}

\begin{abstract}
A political discourse differs from other types of discourses by the double status of the speaker who represents less $\mathrm{him} /$ herself than the community he/she belongs to. This article mainly aims to find out the agentivity and responsibility of the speaker. The analyzed corpus is an interview that took place in the TV news at 8 p.m. of TF1 of 7th March 2016, presented by Gilles Bouleau with Ségolène Royal, the then minister of environment and energy. The debate is about the possible closure of a nuclear plant, in accordance with the commitments of the Socialist Party that was in power. Although it is an interview, the methodology used is not conversational analysis but discourse analysis. The saying and the said of the minister have been analyzed in order to put forward how her discursive ethos is constructed around an ecological problem. Even though she has the legitimacy of speech, of decision and of action, the minister is being rather reluctant in front of a decisive solution. Instead of giving an accurate answer, she chooses for a conditional answer which is highly modal. She delegates her responsibility to others. This is how her discursive ethos appears to be near-agentive or semi-agentive.
\end{abstract}

Keywords: Political Discourse, Discourse Analysis, Ethos, Agentivity. 